\title{
The Impact of COVID-19 on the HIV Care Continuum in a Large Urban Southern Clinic
}

\author{
Jamison Norwood ${ }^{1}\left(\mathbb{0} \cdot\right.$ Asghar Kheshti $^{1} \cdot$ Bryan E. Shepherd $^{2}$ - Peter F. Rebeiro ${ }^{1,2}$ - Aimalohi Ahonkhai ${ }^{1} \cdot$ Sean Kelly ${ }^{1}$. \\ Celestine Wanjalla ${ }^{1}$
}

Accepted: 5 February 2022 / Published online: 23 February 2022

(c) The Author(s), under exclusive licence to Springer Science+Business Media, LLC, part of Springer Nature 2022

\begin{abstract}
Access to care is essential for people with HIV (PWH) but may have been affected during the COVID-19 pandemic. We conducted a retrospective cross-sectional study of adult PWH receiving care in a large southeastern comprehensive care clinic in the United States. Patients in care between January 1, 2017, and July 30, 2020, were included. Race/ethnicity, sex, HIV-1 RNA, CD4 + lymphocyte count were included as baseline covariates. Outcomes included clinic attendance, receipt of HIV-1 RNA PCR testing, and virologic suppression (HIV-1 RNA $<200$ copies $/ \mathrm{mL}$ ); outpatient encounters included new patient encounters, follow-up visits, and mental health encounters. Total medical encounters, including telemedicine, decreased by 827 visits (33\%) when comparing the second quarters of 2019 and 2020. New patient encounters decreased by $23.5 \%$ from 81 to 62 during this period. The second quarter of 2020 saw the lowest number of new patient visits since 2017. HIV-1 RNA testing and the proportion of patients with virologic suppression decreased during the pandemic ( $p<0.001$ for both). Total mental health encounters, on the other hand, increased by 14\% during April-June 2020 compared to April-June 2019. Mental health electronic communications increased by $60 \%$ from 312 to 500 during the same period, with a $20 \%$ increase in medication refills. The COVID-19 pandemic affected outpatient visits, viral load surveillance, and virologic suppression but led to an increase in mental health encounters in a comprehensive care clinic setting.
\end{abstract}

Keywords HIV · COVID-19 · Mental health · Care continuum

\section{Introduction}

COVID-19 has been an ongoing threat to global health since it was first recognized in December $2019[1,2]$. The COVID-19 pandemic has affected our health care system at the national and local levels and has brought light to disparities within medicine and public health. As shelter-inplace orders were issued to mitigate spread, the COVID-19 pandemic also affected our economy and mental health as people were unemployed or underemployed and anxiety

Jamison Norwood

Jamison.norwood@vumc.org

Celestine Wanjalla

celestine.wanjalla@vumc.org

1 Division of Infectious Disease, Department of Medicine, Vanderbilt University Medical Center, A-2200 MCN, 1161 21st Ave S., Nashville, TN 37232-2582, USA

2 Department of Biostatistics, Vanderbilt University, Nashville, TN, USA increased due to social isolation [3]. This has also burdened the healthcare system and led to delays in preventative health care [4]. Importantly, the pandemic has exposed health disparities in African American, Hispanic, and economically disadvantaged communities that have been disproportionately affected by the COVID-19 pandemic $[3,5,6]$.

Linkage to care and sustained retention is essential for people with HIV (PWH) [7]. Sustained retention in care is associated with improved outcomes and durable viral load suppression [8]. While the use of telehealth has been encouraged by the Health Resources and Services Administration (HRSA) Ryan White Program during the COVID-19 pandemic, this remains difficult for many $\mathrm{PWH}$, as access to technology can be limited $[9,10]$. As a result, the COVID19 pandemic has caused decreased access to antiretroviral therapy for some PWH as well as decreased access to HIV prevention services including testing and pre-exposure prophylaxis, which will have long-lasting effects on the Ending the HIV Epidemic initiative by the Department of Health and Human Services [11, 12]. The COVID-19 pandemic has 
highlighted disparities in regards to linkage to care, access to ART, and race/ethnicity within the HIV community [13, 14].

Prior mathematical models have shown the devastating impact that interruptions in care due to COVID-19 can have on mortality for PWH $[15,16]$. However, few studies have evaluated the real-time impact of the COVID-19 pandemic on the HIV continuum of care in a comprehensive care clinic setting. Furthermore, these studies were not coupled with the evaluation of the mental health needs of PWH over the same study period. We assessed the impact that the COVID-19 pandemic has had on the HIV care continuum by evaluating new patient visits, mental health appointments, return visits, and viral load suppression at a large, urban HIV clinic in the southeastern US.

\section{Methods}

\section{Study Population}

We conducted a retrospective observational cross-sectional study of $\mathrm{PWH}>18$ years old receiving care at the Vanderbilt Comprehensive Care Clinic (VCCC), which is an outpatient clinic affiliated with Vanderbilt University Medical Center in Nashville, TN. The clinic provides HIV care throughout Middle Tennessee to more than 3780 patients annually. Providers serve as the primary care provider for the majority of our patients. The patient population is $49 \%$ Caucasian, $43 \%$ African American and 7\% Hispanic; 53\% are economically disadvantaged, defined as an income of fewer than 20,000 dollars per year. Tennessee's stay-at-home order went into effect on March 31, 2020, and expired on April 30, 2020, and we hypothesized that care outcomes would be most impacted during this period. Nonetheless, during that time, the VCCC worked to increase telehealth services to meet the medical and mental health needs of its patients. Telemedicine appointments were made available to all patients on March 17, 2020, using the institution's approved video conferencing software. If video conferencing was unavailable, then a telephone encounter was offered instead. The clinic remained open during the pandemic with at least one provider available for face-to-face encounters. All providers returned to in-person visits by July 27, 2020.

\section{Data Collection and Analysis}

We systematically extracted and validated all laboratory and clinical data from the electronic medical record. Patients were included if they had at least one medical visit at the VCCC between January 1, 2017, and June 30, 2020. Followup was censored at death or at the end of the study period. Race/ethnicity (coded as white non-Hispanic, African American non-Hispanic, other non-Hispanic, and Hispanic) and sex at birth (coded as female or male) were included as risk factors of interest, given the disparate COVID-19 outcomes by these characteristics to date $[6,17]$. Outcomes assessed in each quarter were binary (coded as present or not), and were defined as: (1) attendance at primary care and specialty outpatient clinic visits (including new-patient visits, followup HIV/primary care visits, nutrition visits, and mental health encounters), (2) receipt of HIV-1 RNA PCR testing, (3) viral suppression (HIV-1 RNA $<200$ copies $/ \mathrm{mL}$ ). These variables were chosen to assess the HIV Care Continuum which encompasses a series of HIV-care-related steps from diagnosis to linkage and retention in care to viral suppression [18-20]. These data were systematically abstracted by data managers for the VCCC. Data from the Electronic Medical Record are stored in the Clarity Database ${ }^{\odot}$ and then extracted using Microsoft SQL Server Management Studio $17^{\odot}$. Statistical analysis was performed in GraphPad Prism. Temporal trends and year-to-year changes in the frequency, percentages, and proportions of outcomes were described in the cohort, stratified by baseline risk factors. We compared outcomes in the second quarter of each calendar year in the three years before the pandemic and in 2020. For several comparisons, we chose to compare the second quarter of multiple calendar years to account for secular trends across similar calendar periods year-on-year and assess the impact of the COVID-19 pandemic on care in our clinic during its likely maximal effect, as the COVID-19 state-of-emergency was declared in Nashville just before the second quarter of 2020. Differences in the proportions of patients with viral load testing/suppression by time periods were assessed using chi-square tests; note that these analyses did not account for the correlation between measurements taken from the same individual during time periods. The study was approved by the Institutional Review Board of Vanderbilt University Medical Center.

\section{Results}

There were 1686 medical encounters in the second quarter (April to June) of 2020 compared to 2468, 2560, and 2513 in the second quarters of 2017, 2018, and 2019, respectively. The total number of medical encounters decreased by 827 visits (33\%), including telemedicine, when comparing the second quarter of 2019 to 2020 (Fig. 1). There were similar trends when stratified by sex (Fig. 1) and race/ethnicity (Fig. 1); the second quarter of 2020 showed a similar pattern of decreases in visits by sex and race compared to the second quarter of prior years $(2017,2018,2019)$. Compared to other groups, the VCCC has a smaller fraction of Hispanic and other races, and these visits declined by $23 \%$ (Fig. 1). New-patient encounters in the second quarter decreased by $23.5 \%$ from 2019 to 2020 . Compared to all prior quarters 


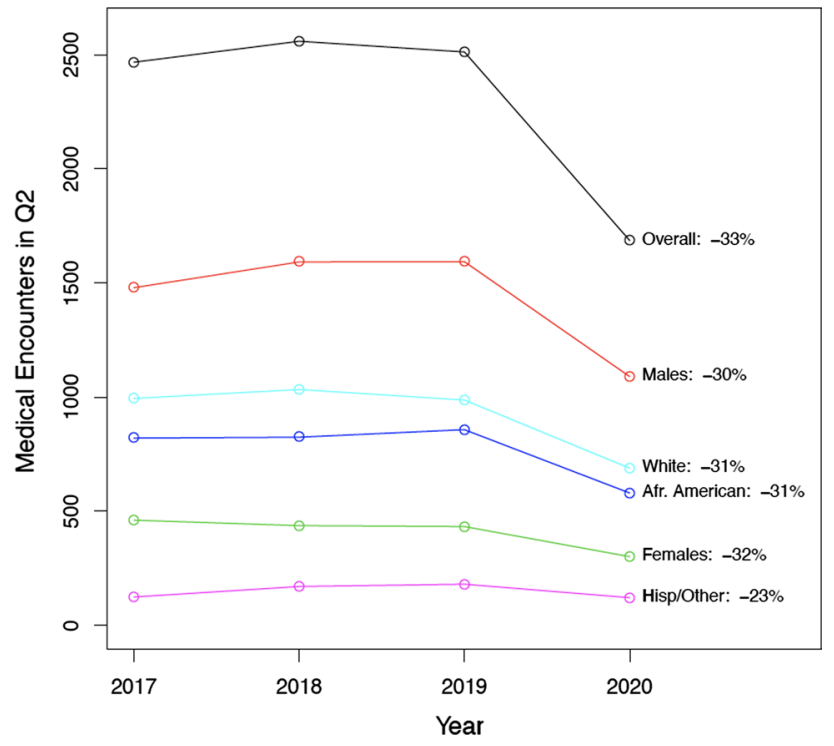

Fig. 1 Total patient encounters decreased in 2020 compared to the identical quarter in 2019. Total second-quarter medical encounters each calendar year stratified by sex and race

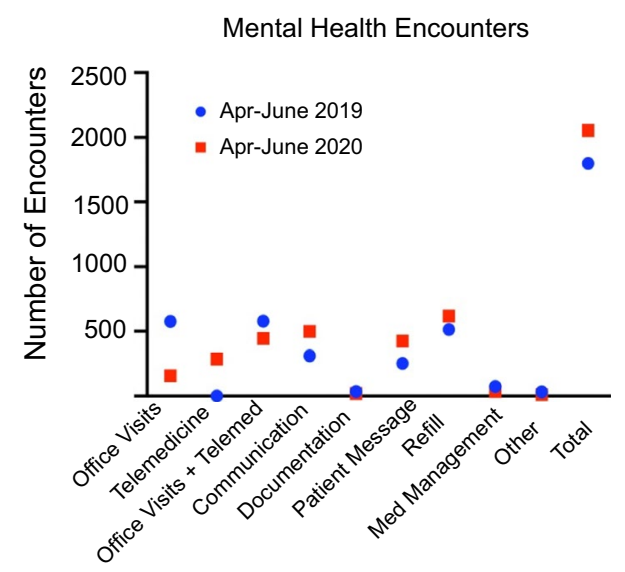

Fig. 2 Total number of mental health encounters in the second quarters of 2019 and 2020. Mental health encounters from April to June 2019 and April-June 2020 (top panel)

from 2017 to the present, the second quarter of 2020 saw the lowest number of new-patient encounters (Data not shown).

Total mental health encounters increased by $14 \%$ from the second quarter of 2019 to 2020 . Mental health telemedicine encounters increased from 2 to 287 while the number of office visits decreased by $73 \%$ from 577 to 158 (Fig. 2). The number of mental health communications (as defined by any formal or informal documentation by the mental health provider in the patient's electronic record) increased by $60 \%$ from 312 to 500 during the same time period (Fig. 2). Patient mental health messages (as defined by electronic messaging between the patient and the mental health provider) increased by $67 \%$ from 254 to 423 (Fig. 2). Medication refills by mental health providers increased by $20 \%$ from 514 to 618 (Fig. 2).

\section{Viral Load Testing and Suppression}

From July 2018 to July 2019, 114 of 3,621 patients (3.1\%) had an outpatient encounter but did not have an HIV-1 RNA PCR obtained. From July 2019 to July 2020, 157 of 3682 patients (4.3\%) had an outpatient encounter but did not have an HIV-1 RNA PCR obtained. Nevertheless, viral suppression was similar when comparing the 12-month periods of 2018-2019 and 2019-2020 (88.2\% and 88.1\%, respectively; $\mathrm{p}=0.92$ ). However, HIV-1 RNA PCR testing and proportions of patients with viral suppression decreased when comparing July to December of 2019, immediately preceding the initial wave of the pandemic, with January to June of 2020 , the one including the initial wave ( $p<0.001$ for both). Of the 3,171 patients with an outpatient visit from July to December 2019, 2902 (91.5\%) had an HIV-1 RNA PCR test and 2644 (83.4\%, or $91.1 \%$ of those tested) were virally suppressed (Fig. 3). In comparison, of the 2736 patients with an outpatient visit from January to June 2020, 2267 (82.9\%) had an HIV-1 RNA PCR test and 2104 (76.9\%, or 92.8\% of those tested) were virally suppressed (Fig. 3). Note that among those who were tested, the proportion with viral suppression slightly increased from July to December 2019 to January-June $2020(p=0.03)$.

\section{Discussion}

Our study demonstrated that the number of outpatient medical encounters among a population of clinically engaged PWH decreased while the number of mental health encounters increased during the initial wave of the COVID-19 pandemic compared to prior years. New patient appointments also decreased during the study period. Our study also found that the proportion of patients without a documented HIV-1 RNA level also increased. These findings provide real-time data showing the impact of the COVID-19 pandemic on HIV care in a large, southeastern HIV clinic.

During our study period, the number of outpatient medical encounters, including new patient visits, decreased while the number of mental health encounters increased. Prior investigations have shown that the COVID-19 pandemic led to clinic closures or interruption in care, with one study demonstrating $82 \%$ of clinics in South Carolina were partially closed or completely closed, which likely accounts for the decreased number of outpatient encounters that we identified [21]. Patients whose appointments were re-scheduled were more likely to have an HIV-1 RNA $<20$ copies/mL compared to those who had a virtual or in-person visit [22]. This suggests that providers may have prioritized patients 
Fig. 3 Viral load testing and suppression. Viral load testing and suppression (Jul-Dec 2019) and (Jan-June 2020). Total number of medical encounters (left panel); encounters with viral load (middle panel) and virally suppressed patients (right panel). $V L$ viral load

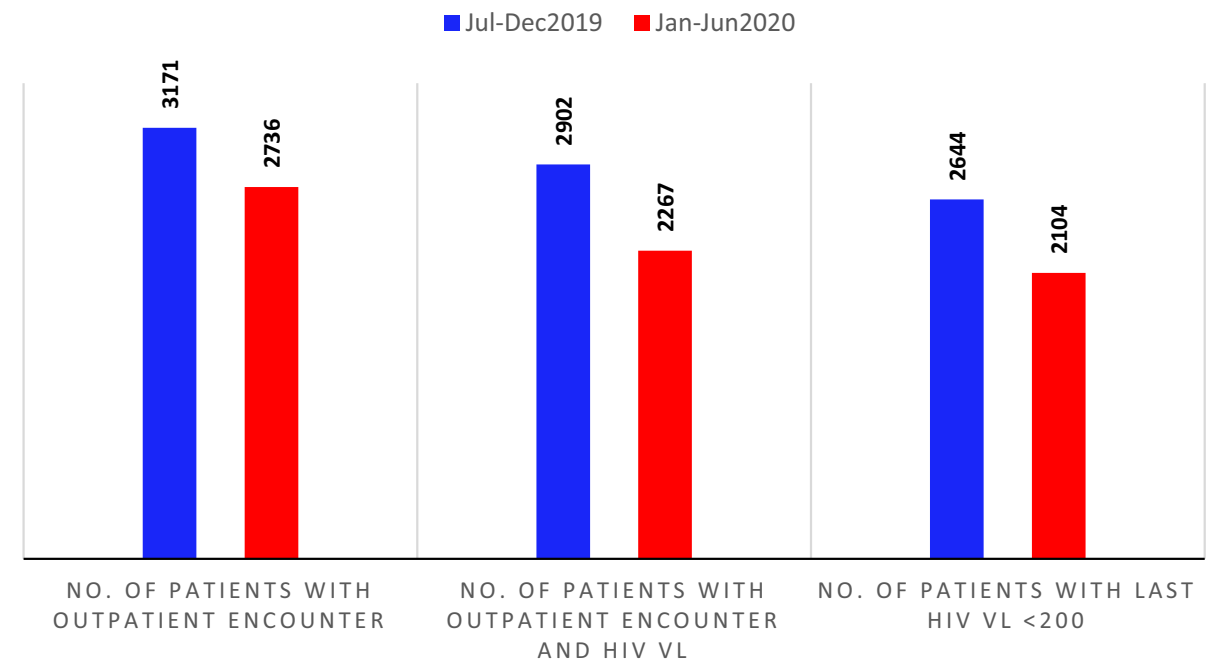

who had uncontrolled HIV or were at higher risk of being lost to follow-up for more frequent and direct contact during the pandemic. While our study demonstrated a decrease in the number of new patient visits, a survey administered to Ryan White Providers revealed that $30 \%$ of respondents reported an increase in the number of new patients [23]. The decrease in new patient appointments at our clinic may be attributed to decreased HIV testing in the community or fewer people moving to our urban area.

Importantly, the number of mental health encounters increased during our study period. It has previously been described that incident stressful events negatively affect medication adherence and virologic suppression among PWH [24]. In our clinic, the psychiatric team contacted patients 2 weeks in advance of their scheduled appointment to convert the visit to a telemedicine or telephone visit to ensure that the technology worked properly and that the patient had access to the technology necessary. Our behavioral health consultant (BHC) extended their services by providing wellness calls to patients identified by their primary care provider (PCP) to be at risk of isolation or mental distress during the pandemic. The BHC was also available to connect into a telemedicine or telephone visit if an immediate or urgent need was identified by the PCP during a telehealth visit. Given the stress of a global pandemic leading to economic and housing instability compounded with increased prevalence of mental illness among PWH [25], HIV clinics should continue to prioritize mental health by increasing access to mental health providers as our patients are likely to experience heightened pandemic-related anxiety, relapse of substance use disorders, and other mental health disorders related to the COVID-19 pandemic [26].

Lastly, our study revealed that fewer patients had an HIV-1 RNA level obtained during the study period. This is likely attributable to increased utilization of telehealth, patient desire to minimize the risk of COVID-19 exposure, and decreased ability to provide testing due to staffing shortages [23]. This is supported by a survey of 1051 US men who have sex with men (MSM) in which $18.8 \%$ reported decreased access to HIV testing; $5.6 \%$ of individuals reported trouble getting an HIV test $[27,28]$. Reductions in HIV testing leads to a delay in HIV diagnosis and engagement in care; therefore, healthcare providers across all specialties must be especially attuned to offering HIV screening to their patients.

There are limitations to our study. First, it is an observational study and there could have been unmeasured confounders. Second, this was a single-site study in Nashville, Tennessee, and may not be generalizable to other populations of PWH. Third, we have limited longitudinal data and it will be important to continue to collect data on the mental health implications as the pandemic continues.

In conclusion, our study showed that the COVID-19 pandemic led to fewer outpatient encounters, including new patient appointments, decreased HIV-1 RNA testing, but an increase in mental health encounters. Therefore, steps should be taken to link and retain patients in care and increase access to mental health providers as the COVID19 pandemic continues.

Author Contributions All authors JN, AK, BES PFR, AA, SK and CW contributed equally to this publication.

Funding This work was funded by the Tennessee Center for AIDS Research Grant P30 AI1 10527. The funding authorities had no role in study design; data collection, analysis, or interpretation; the decision to publish; or preparation of the manuscript.

Data Availability Available.

Code Availability Not applicable. 


\section{Declarations}

Conflict of interest No authors report a conflict of interest.

Ethical Approval The study was approved by the Institutional Review Board of Vanderbilt University Medical Center.

Consent to Participate Not applicable.

Consent for Publication Not applicable.

\section{References}

1. WHO I Novel Coronavirus - China. WHO. http://www.who.int. proxy.library.vanderbilt.edu/csr/don/12-january-2020-novel-coron avirus-china/en/ (2020). Accessed 20 Aug 2020.

2. Huang C, Wang Y, Li X, et al. Clinical features of patients infected with 2019 novel coronavirus in Wuhan, China. Lancet. 2020;395(10223):497-506. https://doi.org/10.1016/S01406736(20)30183-5.

3. Patel JA, Nielsen FBH, Badiani AA, et al. Poverty, inequality and COVID-19: the forgotten vulnerable. Public Health. 2020;183:110-1. https://doi.org/10.1016/j.puhe.2020.05.006.

4. Czeisler MÉ. Delay or avoidance of medical care because of COVID-19-related concerns-United States. MMWR Morb Mortal Wkly Rep. 2020;69:1250. https://doi.org/10.15585/mmwr. mm6936a4.

5. Yancy CW. COVID-19 and African Americans. JAMA. 2020;323(19):1891-2. https://doi.org/10.1001/jama.2020.6548.

6. Hooper MW, Nápoles AM, Pérez-Stable EJ. COVID-19 and racial/ethnic disparities. JAMA. 2020;323(24):2466-7. https:// doi.org/10.1001/jama.2020.8598.

7. Bradley H, Hall HI, Wolitski RJ, et al. Vital signs: HIV diagnosis, care, and treatment among persons living with HIVUnited States, 2011. MMWR Morb Mortal Wkly Rep. 2014;63(47):1113-7.

8. Ulett KB, Willig JH, Lin H-Y, et al. The therapeutic implications of timely linkage and early retention in HIV care. AIDS Patient Care STDS. 2009;23(1):41-9. https://doi.org/10.1089/apc.2008. 0132.

9. Coronavirus Disease 2019 (COVID-19) Frequently Asked Questions. HIV/AIDS Bureau. https://hab.hrsa.gov/coronavirus/frequ ently-asked-questions. (2020). Accessed 28 Sept 2020.

10. Budak JZ, Scott JD, Dhanireddy S, Wood BR. The impact of COVID-19 on HIV care provided via telemedicine-past, present, and future. Curr HIV/AIDS Rep. 2021;18(2):98-104. https://doi. org/10.1007/s11904-021-00543-4.

11. WHO: access to HIV medicines severely impacted by COVID-19 as AIDS response stalls. https://www.who.int/news-room/detail/ 06-07-2020-who-access-to-hiv-medicines-severely-impacted-bycovid-19-as-aids-response-stalls. (2020). Accessed 11 Aug 2020.

12. Giroir BP. The time is now to end the HIV epidemic. Am J Public Health. 2020;110(1):22-4. https://doi.org/10.2105/AJPH.2019. 305380.

13. HIV by Race/Ethnicity I HIV/AIDS I CDC. Published November 14, 2019. https://www.cdc.gov/hiv/group/racialethnic/index.html. (2020). Accessed 11 Aug 2020.

14. Economically Disadvantaged I HIV by Group I HIV/AIDS I CDC. Published December 11, 2019. https://www.cdc.gov/hiv/group/ poverty.html. (2020). Accessed 11 Aug 2020.

15. Lesosky M, Myer L. Modelling the impact of COVID-19 on HIV. Lancet HIV. 2020;7:e596. https://doi.org/10.1016/S23523018(20)30228-9.
16. Mitchell KM, Dimitrov D, Silhol R, et al. The potential effect of COVID-19-related disruptions on HIV incidence and HIV-related mortality among men who have sex with men in the USA: a modelling study. Lancet HIV. 2021;8(4):e206-15. https://doi.org/10. 1016/S2352-3018(21)00022-9.

17. Bienvenu LA, Noonan J, Wang X, Peter K. Higher mortality of COVID-19 in males: sex differences in immune response and cardiovascular comorbidities. Cardiovasc Res. 2020;116(14):2197206. https://doi.org/10.1093/cvr/cvaa284.

18. Grau LE, Brothers S, Kim JY, Khwaja A, Heimer R, Stopka TJ The HIV care continuum in small cities of Southern New England: perspectives of people living with HIV/AIDS, public health experts, and HIV service providers. AIDS Behav. 2021;25(3):897907. https://doi.org/10.1007/s10461-020-03049-z.

19. Uthman OA, Magidson JF, Safren SA, Nachega JB. Depression and adherence to antiretroviral therapy in low-, middle- and highincome countries: a systematic review and meta-analysis. Curr HIV/AIDS Rep. 2014;11(3):291-307. https://doi.org/10.1007/ s11904-014-0220-1.

20. The need for standardisation of the HIV continuum of care ClinicalKey. https://www-clinicalkey-com.proxy.library.vande rbilt.edu/\#!/content/playContent/1-s2.0-S2352301815000867? returnurl=https:\%2F\%2Flinkinghub.elsevier.com $\% 2$ Fretrieve $\%$ 2Fpii\%2FS2352301815000867\%3Fshowall\%3Dtrue\&referrer= https:\%2F\%2Fpubmed.ncbi.nlm.nih.gov\%2F. (2021). Accessed 4 Oct 2021.

21. Qiao S, Li Z, Weissman S, et al. Disparity in HIV service interruption in the outbreak of COVID-19 in South Carolina. AIDS Behav. 2020;25:49. https://doi.org/10.1007/s10461-020-03013-x.

22. Ridgway JP, Schmitt J, Friedman E, et al. HIV care continuum and COVID-19 outcomes among people living with HIV during the COVID-19 pandemic, Chicago, IL. AIDS Behav. 2020;24:2770. https://doi.org/10.1007/s10461-020-02905-2.

23. Delivering HIV Care Prevention in the COVID Era A National Survey of Ryan White Providers - Issue Brief - 9596 I KFF. https://www.kff.org/report-section/delivering-hiv-care-preventionin-the-covid-era-a-national-survey-of-ryan-white-providers-issuebrief/. (2021). Accessed 4 Oct 2021.

24. Mugavero MJ, Raper JL, Reif S, et al. Overload: the impact of incident stressful events on antiretroviral medication adherence and virologic failure in a longitudinal multi-site HIV cohort study. Psychosom Med. 2009;71(9):920-6. https://doi.org/10.1097/PSY. 0b013e3181bfe8d2.

25. Whetten K, Reif S, Whetten R, Murphy-McMillan LK. Trauma, mental health, distrust, and stigma among HIV-positive persons: implications for effective care. Psychosom Med. 2008;70(5):5318. https://doi.org/10.1097/PSY.0b013e31817749dc.

26. Pfefferbaum B, North CS. Mental health and the covid-19 pandemic. N Engl J Med. 2020;383(6):510-2. https://doi.org/10. 1056/NEJMp2008017.

27. Sanchez TH, Zlotorzynska M, Rai M, Baral SD. Characterizing the impact of COVID-19 on men who have sex with men across the United States in April, 2020. AIDS Behav. 2020;24(7):202432. https://doi.org/10.1007/s10461-020-02894-2.

28. DECLINE IN HIV TESTING AND CHANGES IN POSITIVITY RATES DURING THE COVID-19 PANDEMIC. https://www. natap.org/2021/IAS/IAS_109.htm. (2021). Accessed 14 Oct 2021.

Publisher's Note Springer Nature remains neutral with regard to jurisdictional claims in published maps and institutional affiliations. 